\title{
"All hands on deck" - rota-lithotripsy - a combination of rotational atherectomy and intravascular lithotripsy (shockwave) with additional use of a Turnpike Gold microcatheter and guide extension as a novel approach for calcified lesions
}

\author{
Piotr Rola ${ }^{1}$, Adrian Włodarczak ${ }^{2}$, Matuesz Barycki ${ }^{1}$, Jan J. Kulczycki ${ }^{2}$, Barbara Engel ${ }^{1}$, Adrian Doroszko \\ ${ }^{1}$ Department of Cardiology, Provincial Specialized Hospital Legnica, Legnica, Poland \\ ${ }^{2}$ Department of Cardiology, The Copper Health Centre (MCZ) Hospital, Lubin, Poland \\ ${ }^{3}$ Department of Internal Medicine, Hypertension and Clinical Oncology, Wroclaw Medical University, Wroclaw, Poland
}

Adv Interv Cardiol 2021; 17, 2 (64): 214-217

DOI: https://doi.org/10.5114/aic.2021.107503

\section{Introduction}

The progress of the aging process in the general population and increase in the number of comorbidities in elderly people make the success of interventional cardiology treatments increasingly challenging. Alongside increased lifespan, we observe a growing number of complex percutaneous coronary interventions ( $\mathrm{PCl}$ ) performed in everyday practice. The procedures are inextricably associated with increased prevalence of patients with heavily calcified lesions which may affect the periprocedural complication and revascularization failure rate and - as a result - the short- and long-term survival [1]. Additionally, unfavorable coronary anatomy (tortuosity, angulation, presence of major side branch, and excessive calcium deposits) commonly complicate the use of sophisticated maneuvers and dedicated technologies to perform a successful $\mathrm{PCl}$.

Atherectomy devices have been widely used as safe and efficient tools to modify plaque calcium burden plaque [2]. Nevertheless, rotational atherectomy (RA) is associated with a substantial risk of complications such as perforation, short-term closure, side branch loss, and the slow-flow/no-reflow phenomenon, vasospasm, and burr entrapment [3] and requires appropriate operator's training.

A recent study [4] suggested that shockwave intravascular lithotripsy (S-IVL) might be a simple, safe, and effective alternative method aiming at modification of heavily calcified lesions. The Shockwave Medical Intra- vascular Lithotripsy System (Shockwave Medical Inc., Fremont, United States) delivers sonic pressure waves during low-pressure balloon inflation, which affects mainly calcifications, leading to fragmentation of calcium nodules.

In this paper we present a novel method - rota-lithotripsy - which combines the two plaque modification techniques RA and S-IVL with the additional use of uncustomary maneuvers and hardware, to deal with an undilatable calcified lesion.

\section{Case report}

The case concerns a 62-year-old male, active cigarette smoker with hypertension, hyperlipidemia, chronic mild anemia, chronic kidney disease on hemodialysis, with lower extremity peripheral artery disease (PAD) after surgical revascularization (left femoral $\rightarrow$ popliteal artery bypass), with active ulcer of the left foot, history of myocardial infarction without ST-segment elevation (NSTEMI) treated percutaneously with a bare metal stent (BMS) to the right coronary artery (RCA), and in subsequent scheduled BMS implantation to the left anterior descending artery (LAD) and the circumflex (Cx), 11 years prior to the current hospitalization. He had a history of NSTEMI 1 year previously treated with implantation of drug eluting stents (DES) to the proximal part of the RCA and an unsuccessful attempt of crossing a lesion in the distal part of the RCA (due to heavily calcification in the body of the lesion the patient was qualified for optimal medical therapy of CAD). 
He was admitted to hospital with chest pain (class IV in the CCS scale) ongoing for $2 \mathrm{~h}$. An electrocardiogram showed sinus rhythm $80 /$ min conducted with the right bundle branch block (RBBB) and the serum high-sensitivity troponin-I was positive. Coronary angiogram revealed: chronic total occlusion of the LAD with two coexisting culprit lesions in the RCA (proximal in-stent restenosis and distal highly calcified subtotal lesion) (Figure $1 \mathrm{~A}$ ). Accumulated Syntax Score was 39.5 points. Due to advanced atherosclerosis, comorbidities and ongoing ischemic symptoms a rescue $\mathrm{PCl}$ was performed by the right femoral approach using the AL 1.0 Guide-Catheter (7F)
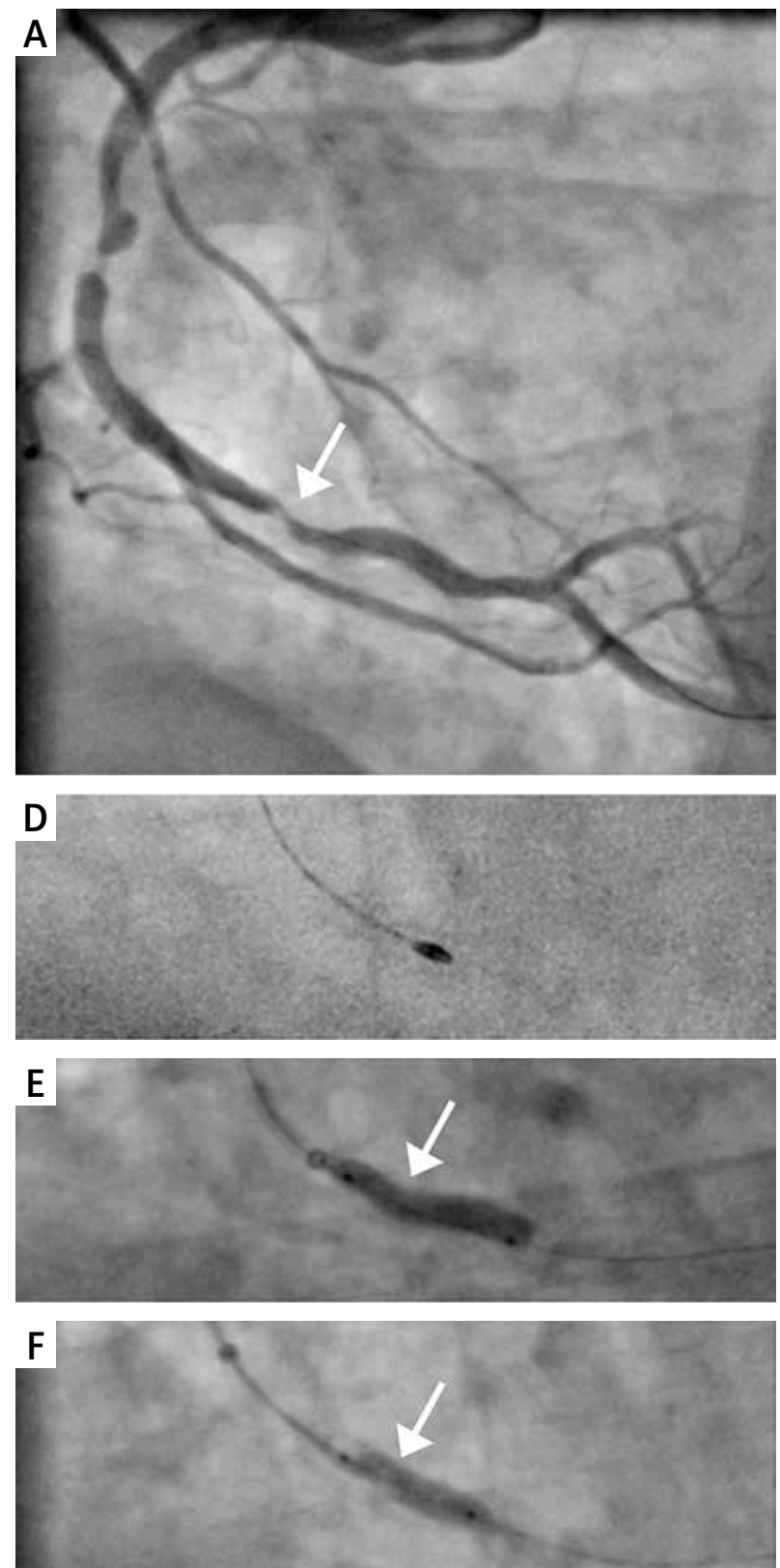

(Medtronic Ireland, Galway, Ireland). We managed to cross the distal lesion with a Choice ES guidewire (Boston Scientific, Marlborough, United States). Despite use of the 7F guiding extension TrapLiner (Teleflex Incorporated, Wayne, United States) all attempts to cross the lesion with the FineCross microcatheter (Terumo Corporation, Tokyo, Japan) and a low-profile balloon Ryurei $1.0 \times 10 \mathrm{~mm}$ (Terumo) failed. The successful crossing was achieved using an additional support by TrapLiner (Teleflex) and Turnpike Gold 135 (Vascular Solutions LLC, Minneapolis, United States) (Figure 1 B) - a microcatheter with a threaded tip, providing rotational advance-
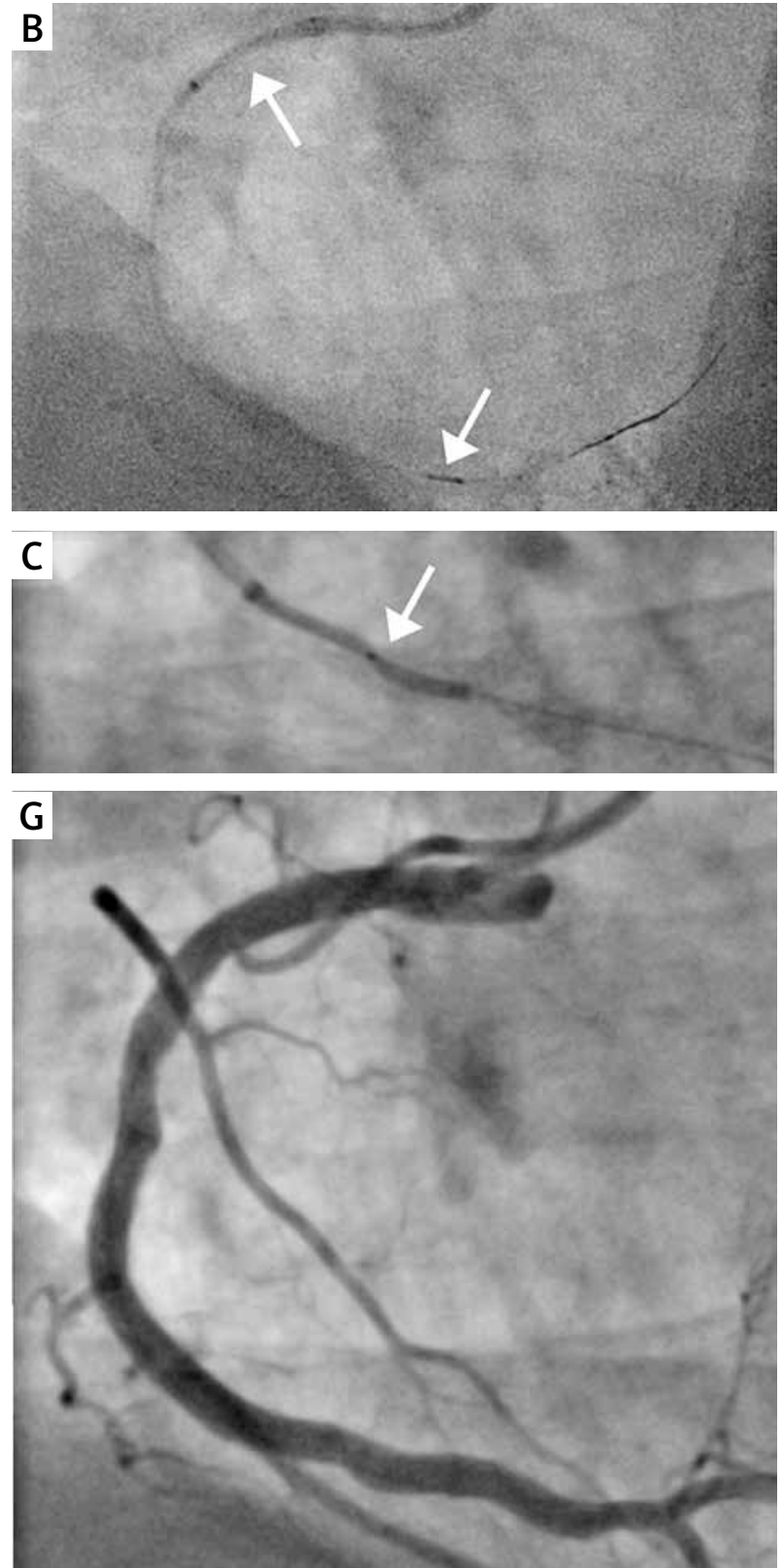

Figure 1. A - Coronary angiography of the right coronary artery - two culprit lesions, B - successful lesion crossing with TrapLiner and Turnpike, C - underexpansion of $1.5 \times 20 \mathrm{~mm}$ balloon catheter, $\mathbf{D}$ - rotational atherectomy with $1.75 \mathrm{~mm}$ burr, $\mathbf{E}$ - underexpansion of $3.0 \times 15 \mathrm{~mm}$ non-compliant balloon catheter, $\mathbf{F}-$ full expansion of intravascular lithotripsy $3.5 \times 12 \mathrm{~mm}$ catheter, $\mathbf{G}-$ final angiographic result 
ment when rotated clockwise. In the next step we performed ineffective predilation with Ryurei (Terumo) $1.5 \times$ $20 \mathrm{~mm}$. Significant underexpansion of the balloon catheter at $18 \mathrm{~atm}$ was observed (Figure $1 \mathrm{C}$ ). Therefore, we exchanged the guidewire on the Rotawire-Extra-Support (Boston Scientific) and performed successful rotational atherectomy (RA) with the ROTAPRO Rotational Atherectomy System (Boston Scientific) using a Rotablator burr size of $1.75 \mathrm{~mm}$ (Figure $1 \mathrm{D}$ ). Despite lesion preparation with the RA, we still observed a significant "dogbone effect" on the non-compliant (NC) Emerge (Boston Scientific) balloon $3.0 \times 15 \mathrm{~mm}$ (22 atm) (Figure $1 \mathrm{E})$. Hence, we performed the S-IVL using a $3.5 \times 12 \mathrm{~mm}$ catheter. Delivery of the device was facilitated by use of a guiding extension. After application of 40 ultrasonic pulses, full expansion was obtained (Figure $1 \mathrm{~F}$ ). Implantation of three overlapping drug eluting stents - Resolute ONYX (Medtronic) $3.5 \times 38 \mathrm{~mm}, 4.0 \times 38 \mathrm{~mm}$ and $4.0 \times 34 \mathrm{~mm}$ (16 atm) - was followed by a $4.0 \times 20 \mathrm{~mm}$ (22 atm) NC Emerge (Boston Scientific) postdilation. Finally, we obtained a satisfactory angiographic result (Figure $1 \mathrm{G}$ ). On the $7^{\text {th }}$ day after the procedure, the patient was discharged. Follow-up transthoracic echocardiography showed an enlarged hypokinetic left ventricle with coexisting thinning and lack of vitality related to the anterior wall, interventricular septum and apex (LVEDd/LVESd = $64 \mathrm{~mm} / 50 \mathrm{~mm}$ ) with reduced ejection fraction (EF 30\%) and coexisting moderate functional mitral regurgitation, without other mechanical complications. Due to the previously observed analogical impairment of myocardial contractility, ICD implantation was scheduled.

\section{Discussion}

To the best of our knowledge, we are the very first to present a well-documented successful application of a complex advanced plaque-modifying method - RA and S-IVL application combined with the use of a wide range of hardware, such as a threaded-tip microcatheter and guide-extension catheter in a subject with acute coronary syndrome (ACS). So far, in the literature, we can find only a few case reports $[5,6]$ of combined rotational atherectomy and intravascular lithotripsy. Both mentioned methods require crossing the lesion with a guidewire, which might be challenging in a calcified subtotal lesion especially in the case of Rotawire (Boston Scientific), which is difficult to control and susceptible to fracture. In order to avoid destruction, numerous operators exchange the guidewires using a microcatheter. However, in the present case, despite use of a low profile microcatheter (FineCross), we were unable to cross the lesion even with the additional support of a guiding extension (TrapLiner) along with aggressive guiding (AL 1.0 7F). Therefore, we decided to use the TrapLiner with Turnpike Gold catheter, which is constructed with a unique multi-layer shaft that provides improved flexibility, torque and tracking over the guidewire [7]. Recently we proved that it can facilitate overcoming a very calcified CTO lesion [8]. Rotational atherectomy is limited to superficial plaque modification and exerts no effect on deeper calcium deposits. Hence, unlike the bulky S-IVL catheter, it can modify the body of a tight subcritical lesion. However, in this case - despite the use of adequate burr sizing (burr-to-artery ratio of 0.5 to 0.6 [9]) - the postdilatation with an NC balloon failed. An aggressive strategy with large burrs (burr/artery ratio $>0.7$ ) to achieve maximum debulking is associated with higher rates of periprocedural complications [10] and often requires an increased diameter of the guiding system (which can lead to vascular access-related complication). Hence, it is an unfavorable option for a patient with PAD, especially in the ACS setting. To solve these issues, additional use of the S-IVL system was applied. It is a novel balloon catheter-based coronary system for IVL, which converts electrical energy into mechanical energy (acoustic wave with high-pressure amplitude) during low-pressure balloon inflation. Unlike RA, which "pulverizes" the superficial part of calcified deposits, S-IVL, thanks to the physical property of sonic pulses, leads to defragmentation of an entire cross-section of calcium nodules, also affecting the inner part of calcified deposits. It allows one to treat lesions resistance to "classical" atheroablation methods (deep calcium deposit or significant stent underexpansion) [11]. However, the low crossing profile of the IVL catheter device often leads to difficulties in advancing the Shockwave balloon into the body of critical calcified lesions. Therefore to perform successful $\mathrm{PCl}$ additional use of initial plaque modification methods is often mandatory. In the present case we were forced to prepare the lesion with RA. It should be emphasized that due to severe artery tortuosity and coexisting severe calcification, strong support from a guide catheter with additional use of a guiding extension system seems to be crucial at each part of the procedure (even after successful passage of the Rota burr). There are some data [12] suggesting that this hardware can also facilitate performing RA. Although in the described case, endovascular imaging was not used, it might be a valuable source of information allowing for: an insightful assessment of the cause of previously implanted stents' failure (in-stent restenosis or neoatherosclerosis), detailed analyses of calcium structure in the culprit lesion, and more accurate optimization of currently implanted stents.

\section{Conclusions}

Overcoming the difficulties associated with highly complex $\mathrm{PCl}$ in a calcified lesion often requires multiple specialized devices. They should be considered not as a separate technique but rather as complementary to each other. We have described a novel technique of overcoming heavily calcified lesions, using a broad range of hardware, thanks to applying its specific properties. 


\section{Conflict of interest}

The authors declare no conflict of interest.

\section{References}

1. Sharma SK, Bolduan RW, Patel MR, et al. Impact of calcification on percutaneous coronary intervention: MACE-Trial 1-year results. Catheter Cardiovasc Interv 2019; 94: 187-94.

2. Danek BA, Karatasakis A, Karacsonyi J, et al. A meta-analysis of contemporary lesion modification strategies during percutaneous coronary intervention in 244,795 patients from 22 studies. J Invasive Cardiol 2017; 29: E167-76.

3. Tomey MI, Kini AS, Sharma SK. Current status of rotational atherectomy. JACC Cardiovasc Interv 2014; 7: 345-53.

4. Ali ZA, Nef H, Escaned J, et al. Safety and effectiveness of coronary intravascular lithotripsy for treatment of severely calcified coronary stenoses: the disrupt CAD II study. Circulation Cardiovasc Interv 2019; 12: e008434.

5. Aznaouridis K, Bonou M, Masoura C, et al. Rotatripsy: a hybrid" drill and disrupt" approach for treating heavily calcified coronary lesions. J Invasive Cardiol 2020; 32: E175.

6. Jurado-Román A, Gonzálvez A, Galeote G, et al. RotaTripsy: combination of rotational atherectomy and intravascular lithotripsy for the treatment of severely calcified lesions. JACC CardiovasC Interv 2019; 12: e127-9.

7. Mishra S. Language of CTO interventions - focus on hardware. Indian Heart J 2016; 68: 450-63.

8. Kulczycki J, Włodarczak A, Łanocha $M$, et al. Tough calcification versus tough cardiologist: a case report. Kardiol Pol 2021; 79: 354-55.

9. Kubler P, Zimoch W, Kosowski M, et al. Outcomes and clinical predictors of mortality of patients treated with rotational atherectomy: a single center registry. Adv Interv Cardiol 2016; 12: 193-4.

10. Safian RD, Feldman T, Muller DW, et al. Coronary angioplasty and Rotablator atherectomy trial (CARAT): immediate and late results of a prospective multicenter randomized trial. Catheter Cardiovasc Interv 2001; 53: 213-20.

11. Brinton TJ, Ali ZA, Hill JM, et al. Feasibility of shockwave coronary intravascular lithotripsy for the treatment of calcified coronary stenoses. Circulation 2019; 139: 834-6.

12. Pawłowski T, Śliwiński Z, Modzelewski P, et al. Guiding catheter extension facilities high speed rotational atherectomy for distal right coronary artery lesion. Adv Interv Cardiol 2020; 16: 507-8. 